Korean J. Math. 20 (2012), No. 4, pp. 533-540

http://dx.doi.org/10.11568/kjm.2012.20.4.533

\title{
EXISTENCE OF THE SOLUTIONS FOR THE ELLIPTIC PROBLEM WITH NONLINEAR TERM DECAYING AT THE ORIGIN
}

\author{
Q-Heung Choi and Tacksun Jung*
}

\begin{abstract}
We consider the multiplicity of the solutions for the elliptic boundary value problem with $C^{1}$ nonlinear term decaying at the origin. We get a theorem which shows the existence of the nontrivial solution for the elliptic problem with $C^{1}$ nonlinear term decaying at the origin. We obtain this result by reducing the elliptic problem with the $C^{1}$ nonlinear term to the elliptic problem with bounded nonlinear term and then approaching the variational method and using the mountain pass geometry for the reduced the elliptic problem with bounded nonlinear term.
\end{abstract}

\section{Introduction}

Let $\Omega$ be a bounded domain in $R^{n}$ with smooth boundary $\partial \Omega$. Let $g: \bar{\Omega} \times R \rightarrow R$ be a $C^{1}$ function. In this paper we consider the following elliptic problem with Dirichlet boundary condition

Received November 10, 2012. Revised December 13, 2012. Accepted December $15,2012$.

2010 Mathematics Subject Classification: 35J20, 35J25.

Key words and phrases: Elliptic boundary value problem, nonlinear term decaying at the origin, bounded nonlinear term, variational method, critical point theory, mountain pass geometry, (P.S. $)_{c}$ condition.

This work (Tacksun Jung) was supported by Basic Science Research Program through the National Research Foundation of Korea(NRF) funded by the Ministry of Education, Science and Technology (KRF-2010-0023985).

*Corresponding author.

(C) The Kangwon-Kyungki Mathematical Society, 2012.

This is an Open Access article distributed under the terms of the Creative commons Attribution Non-Conercial License (http://creativecommons.org/licenses/by$\mathrm{nc} / 3.0 /$ ) which permits unrestricted non-commercial use, distribution and reproduction in any medium, provided the original work is properly cited. 


$$
\begin{array}{rlr}
-\Delta u & =g(x, u(x)) \quad \text { in } \Omega, \\
u & =0 \quad \text { on } \partial \Omega . &
\end{array}
$$

We assume that $g$ satisfies the following:

(g1) $g \in C^{1}(\bar{\Omega} \times R, R)$,

$(g 2) g(x, 0)=0, g(x, \xi)=o(|\xi|)$ uniformly with respect to $x \in \bar{\Omega}$,

(g3) there exists $\xi \geq 0$ such that $g(x, \xi) \leq 0 \forall x \in \bar{\Omega}$,

(g4) there exist a constant $r>0$, a sphere $S_{r}$ with radius $r$ and an element $e$ such that $e \in H \backslash S_{r}, e<\xi$ and $g(x, e)>0$.

This type boundary value problem was considered by many authors (cf. [1], [3], [4], [5], [8]). Rabinowitz [8] showed that if $g \in C^{1}(\bar{\Omega}, R)$, $g$ is of the form $g(x, t)=\lambda a(x) t+p(x, t), p$ satisfies $p(\xi)=o(|\xi|)$ as $\xi \rightarrow 0, a(x)$ is continuous and $a(x)>0$ in $\bar{\Omega}$, then (1.1) has several kinds of solutions under some additional assumptions. He proved this result by the critical point theory and the variational method. Chang [1] also proved that if $g(x, t)=\lambda(a(x) t+p(x, t))$, then (1.1) has at least $k$ solutions under the conditions $(g 1)^{\prime}-g(3)^{\prime}$

$(g 1)^{\prime}$ There exists $\xi \geq 0$ such that $a(x) \xi+p(\xi) \leq 0 \forall x \in \bar{\Omega}$,

$(g 2)^{\prime} p \in C^{1}(\bar{\Omega}, R), p$ satisfies $p(\xi)=o(|\xi|)$ as $\xi \rightarrow 0, a(x)$ is continuous on $\bar{\Omega}$ and $a(x)>0$, $(g 3)^{\prime} p(-\xi)=-p(\xi)$.

He proved this result by the critical point theory. In $[6,7]$ the authors investigated the existence of multiple solutions of the elliptic boundary value problems.

The eigenvalue problem

$$
\begin{gathered}
\Delta u+\lambda u=0 \quad \text { in } \Omega, \\
u=0 \quad \text { on } \partial \Omega
\end{gathered}
$$

has infinitely many eigenvalues $\lambda_{j}, j \geq 1$, which are repeated as often as its multiplicity, and the corresponding eigenfunctions $\phi_{j}, j \geq 1$, suitably normalized with respect to $L^{2}(\Omega)$ inner product.

Let $H$ be a Sobolev space with a norm

$$
\|u\|^{2}=\int_{\Omega}|\nabla u|^{2} d x
$$


We are looking for the weak solution of (1.1), i.e.,

$$
\int_{\Omega}[\nabla u \cdot \nabla v-g(x, u) v] d x=0 \quad \text { for all } \quad v \in H .
$$

We consider the associated functional of (1.1)

$$
I(u)=\int_{\Omega}\left[\frac{1}{2}|\nabla u|^{2}-G(x, u)\right] d x,
$$

where $G(s)=\int_{0}^{s} g(x, \tau) d \tau$. By $(g 1), I$ is well defined.

Our main result is the following.

Theorem 1.1. Assume that $g$ satisfies the conditions $(g 1)-(g 4)$. Then (1.1) has at least one nontrivial weak solution.

We prove Theorem 1.1 by reducing the elliptic problem (1.1) to the elliptic problem with bounded nonlinear term and then approaching the variational method and using the mountain pass geometry for the reduced elliptic problem with bounded nonlinear term. The outline of the proof of Theorem 1.1 is as follows: In section 2, we prove that functional $I(u) \in C^{1}$ and the reduced functional $\hat{I}$ of $I$ satisfies the Palais Smale condition by the maximum principle. In section 3 , we show that the reduced functional $\hat{I}$ satisfies the mountain pass geometry and so prove that $\hat{I}$ has at least one nontrival critical point, from which we prove Theorem 1.1.

\section{Reduction to the elliptic problem with bounded nonlinear term}

Lemma 2.1. Assume that $g$ satisfies $(g 1)-(g 3)$. Then $I(u)$ is continuous and Fréchet differentiable in $H$ with Fréchet derivative

$$
\nabla I(u) h=\int_{\Omega}[\nabla u \cdot \nabla h-(g(x, u)) h] d x \quad \text { for all } h \in H .
$$

If we set

$$
F(u)=\int_{\Omega} G(x, u) d x
$$

then $F^{\prime}(u)$ is continuous with respect to weak convergence, $F^{\prime}(u)$ is compact, and

$$
F^{\prime}(u) h=\int_{\Omega} g(x, u) h d x \quad \text { for all } h \in H,
$$


this implies that $I \in C^{1}(H, R)$ and $F(u)$ is weakly continuous.

The proof of Proposition 3.1 has the similar process to that of the proof in Appendix $B$ in [2].

Now we shall reduce the problem (1.1) to the problem with bounded nonlinear term.

Lemma 2.2. Assume that $g$ satisfies the conditions $(g 1)-(g 4)$. Let

$$
\hat{g}(x, t)= \begin{cases}g(x, \xi) & \text { if } t>\xi, \\ g(x, t) & \text { if } t \leq \xi\end{cases}
$$

Assume that $u \in H$ is a solution of the equation

$$
\left\{\begin{array}{l}
-\Delta u(x)=\hat{g}(x, u(x)) \quad x \in \Omega, \\
\left.u\right|_{\partial \Omega}=0 .
\end{array}\right.
$$

Then $u \leq \xi$, so $u$ is a solution of (1.1).

Proof. By the standard regularity theorem $u \in W_{p}^{2}(\Omega) \forall p<+\infty$, so $u \in C^{1}(\bar{\Omega})$. Let us set

$$
C=\{x \in \Omega \mid u(x)>\xi\} .
$$

Then we have

$$
\left\{\begin{array}{l}
-\Delta u(x) \leq 0 \quad \forall x \in C, \\
\left.u\right|_{\partial C} \leq \xi
\end{array}\right.
$$

By the maximum principle, we have

$$
u(x)<\xi \quad \text { in } C
$$

so $C=\emptyset$. Thus $u(x) \leq \xi$.

By Lemma 2.2, it suffices to show the existence of at least one nontrivial solution of (2.2) to prove the existence of at least one nontrivial solution of (1.1). Now we shall show that (2.2) has at least one nontrivial solution by approaching the variational method and applying mountain pass theorem in the critical point theory. By Lemma 2.1, the associated functional of $(2.2)$

$$
\hat{I}(u) \in C^{1}(H, R) .
$$

First we shall show that

$$
\hat{I}(u)=\int_{\Omega}\left[\frac{1}{2}|\nabla u|^{2}-\hat{G}(x, u)\right] d x,
$$

where $\hat{G}(x, s)=\int_{0}^{s} \hat{g}(x, \tau) d \tau$, satisfies Palais-Smale condition. 
Lemma 2.3. Assume that $g$ satisfies the conditions $(g 1)-(g 3)$. Then the functional $\hat{I}$ satisfies Palais-Smale condition: Any sequence $\left(u_{m}\right)$ in $H$ for which $\left|\hat{I}\left(u_{m}\right)\right| \leq M$ and $\hat{I}^{\prime}\left(u_{m}\right) \rightarrow 0$ as $m \rightarrow \infty$ possesses a convergent subsequence.

Proof. Any sequence $\left(u_{m}\right)$ in $H$ such that $\left|\hat{I}\left(u_{m}\right)\right| \leq M$ and $\hat{I}^{\prime}\left(u_{m}\right) \rightarrow$ 0 as $m \rightarrow \infty$. we shall show that $\left(u_{m}\right)$ has a convergent subsequence. It suffices to show that $\left(u_{m}\right)$ is bounded. We claim that $u_{m} \leq \xi$. By contradiction, we suppose that $u_{m}>\xi$. Letting $w_{m}=\frac{u_{m}}{\left\|u_{m}\right\|}$, we have

$$
\begin{aligned}
& 0 \longleftarrow \frac{\hat{I}^{\prime}\left(u_{m}\right) u_{m}}{\left\|u_{m}\right\|^{2}} \\
& =\int_{\Omega}\left[-\Delta w_{m} \cdot w_{m}-\frac{\hat{g}\left(x, u_{m}\right) u_{m}}{\left\|u_{m}\right\|^{2}}\right] d x .
\end{aligned}
$$

Since $\left\|w_{m}\right\|=1$, the sequence $\left(w_{m}\right)$ has a convergent subsequence, up to a subsequence, $\left(w_{m}\right)$ which converges weakly to $w$. Letting $m \rightarrow \infty$ in (2.4), we have

$$
0=\int_{\Omega}\left[\left[-\Delta w \cdot w-\lim _{m \rightarrow \infty} \frac{\hat{g}\left(x, u_{m}\right)}{\left\|u_{m}\right\|} w\right] d x .\right.
$$

We note that since $\hat{g}\left(x, u_{m}\right)$ is bounded and $u_{m}>\xi, \frac{\hat{g}\left(x, u_{m}\right)}{\left\|u_{m}\right\|}$ is bounded and $\lim _{m \rightarrow \infty} \frac{\hat{g}\left(x, u_{m}\right)}{\left\|u_{m}\right\|}$ exists. It follows from (2.5) that

$$
\begin{aligned}
-\Delta w & =\lim _{m \rightarrow \infty} \frac{\hat{g}\left(x, u_{m}\right)}{\left\|u_{m}\right\|} \quad \text { in } H, \\
w & =0 \quad \text { on } \partial \Omega .
\end{aligned}
$$
then

$$
\frac{\hat{g}\left(x, u_{m}\right)}{\left\|u_{m}\right\|}=\frac{g(x, \xi)}{\left\|u_{m}\right\|} .
$$

Letting $m \rightarrow \infty$ in (2.6), we have $\lim _{m \rightarrow \infty} \frac{\hat{g}\left(x, u_{m}\right)}{\left\|u_{m}\right\|} \leq 0$. It follows from (2.6) and (2.7) that

$$
\begin{gathered}
-\Delta w \leq 0 \quad \forall x \in D, \\
\left.w\right|_{\partial D} \leq \lim _{m \rightarrow \infty} \frac{\xi}{\left\|u_{m}\right\|} .
\end{gathered}
$$


By Maximum principle, we have $w \leq \lim _{m \rightarrow \infty} \frac{\xi}{\left\|u_{m}\right\|}$ in $D$, and hence $D=\emptyset$. That is, $w \leq \lim _{m \rightarrow \infty} \frac{\xi}{\left\|u_{m}\right\|}$, which is impossible because $w_{n}>$ $\frac{\xi}{\left\|u_{m}\right\|}$. Thus we have $u_{m} \leq \xi$. Thus the sequence $\left(u_{m}\right)$ has a convergent subsequence, up to a subsequence, which converges weakly to $u$. Thus we have

$$
\begin{gathered}
\int_{\Omega}\left[-\Delta u_{m} \cdot u_{m}-\hat{g}\left(x, u_{m}\right) u_{m}\right] d x=\left\|u_{m}\right\|^{2}-\int_{\Omega} \hat{g}\left(x, u_{m}\right) u_{m} d x \\
\longrightarrow \int_{\Omega}[-\Delta u \cdot u-\hat{g}(x, u) u] d x=\|u\|^{2}-\int_{\Omega} \hat{g}(x, u) u d x
\end{gathered}
$$

Since $u_{m}$ is bounded, $\hat{g}\left(x, u_{m}\right)$ is bounded and $g(1)$ holds,

$$
\lim _{m \rightarrow \infty} \int_{\Omega} \hat{g}\left(x, u_{m}\right) u_{m} d x=\int_{\Omega} \hat{g}(x, u) u d x .
$$

Thus $\lim _{m \rightarrow \infty}\left\|u_{m}\right\|=\|u\|$, so the sequence $\left(u_{m}\right)$ converges strongly to $u$. Thus $\hat{I}$ satisfies the Palais-Smale condition.

\section{Proof of Theorem 1.1}

We shall show that the functional $\hat{I}$ satisfies the mountain pass geometry. Let

$$
B_{R}=\{u \in H \mid\|u\| \leq R\}
$$

and

$$
S_{R}=\{u \in H \mid\|u\|=R\} .
$$

Lemma 3.1. Assume that $g$ satisfies the conditions $(g 1)-(g 4)$. Then

(i) there exist constants $\rho, \alpha>0$ such that $\rho<r$ ( $r$ is a constant in $(g 4)), \inf _{u \in S_{\rho}} \hat{I}(u) \geq \alpha$

(ii) there is an $e \in H \backslash S_{\rho}$ such that $\hat{I}(e) \leq 0$.

Proof. (i) Let $u \in H$. By $(g 2)$ and ( $g 4)$, there exist a small number $a>0, \rho>0$ and a sphere $S_{\rho}$ with the radius $\rho$ such that $\rho<r, a<\frac{1}{2} \lambda_{1}$ and $|\hat{G}(x, u)| \leq a\|u\|^{2}$ if $u \in S_{\rho}$. If we choose $u \in S_{\rho}$, then there exists a constant $\alpha>0$ such that 


$$
\begin{aligned}
\hat{I}(u) & =\int_{\Omega}\left[-\frac{1}{2} \Delta u \cdot u-\hat{G}(x, u(x))\right] d x \\
& \geq \frac{1}{2} \lambda_{1}\|u\|_{\Omega}^{2}-a\|u\|^{L^{2}(\Omega)}=\left(\frac{1}{2} \lambda_{1}-a\right)\|u\|^{2} \geq \alpha .
\end{aligned}
$$

(ii) Let us choose an element $u \in H \backslash S_{\rho}$ such that $u \leq \xi$. Since the operator $-\Delta$ is positive operator, $-\Delta u \leq-\Delta \xi$. Then we have

$$
\begin{aligned}
\hat{I}(u) & =\int_{\Omega}[-\Delta u \cdot u-\hat{G}(x, u)] d x \leq \int_{\Omega}[-\Delta \xi \cdot \xi-\hat{G}(x, u)] d x \\
& =-\int_{\Omega} \hat{G}(x, u) d x .
\end{aligned}
$$

By $(g 4)$, there exist a constant $r>0$ and an element $e \in H \backslash S_{\rho}$ such that $r>\rho, e<\xi$ and $G(x, e)>0$. Then

$$
\hat{I}(e) \leq-\int_{\Omega} \hat{G}(x, e)=-\int_{\Omega} G(x, e)<0 .
$$

Thus (ii) is proved.

\section{Proof OF Theorem 1.1}

We will show that $\hat{I}(u)$ has a nontrivial critical point by the mountain pass theorem. By Lemma 2.1, $\hat{I}(u)$ is continuous and Fréchet differentiable in $H$. By Lemma 2.3, the functional $\hat{I}$ satisfies (PS) condition. We note that $I(0)=0$. By Lemma 3.1, there exist constants $\rho, \alpha>0$ such that $\rho<r, \inf _{u \in S_{\rho}} \hat{I}(u) \geq \alpha$, and there is an $e \in H \backslash S_{\rho}$ such that $\hat{I}(e)<0$. Let us set

$$
\Gamma=\{\gamma \in C([0,1], H) \mid \gamma(0)=0, \gamma(1)=e\} .
$$

By the mountain pass theorem, $\hat{I}$ possesses a critical value $c \geq \alpha$ characterized as

$$
c=\inf _{\gamma \in \Gamma} \max _{u \in \text { gamma }} I(u) .
$$

Thus we prove that $\hat{I}$ has at least one nontrivial critical point, so (2.2) has at least one nontrivial weak solution. By lemma 2.2, this solution of (2.2) is also a weak solution of (1.1). Thus (1.1) has at least one nontrivial weak solution, and hence we prove Theorem 1.1. 


\section{References}

[1] K.C. Chang, Infinite dimensional Morse theory and multiple solution problems, Birkhäuser, (1993).

[2] Q.H. Choi, and T. Jung, Multiplicity of solutions and source terms in a fourth order nonlinear elliptic equation, Acta Math. Sci. 19 (4) (1999), 361-374.

[3] T. Jung and Q.H. Choi, Multiple solutions result for the mixed type nonlinear elliptic problem, Korean J. Math. 19 (4) (2011), 423-436.

[4] T. Jung and Q.H. Choi, Bifurcation problem for the superlinear elliptic operator, Korean J. Math. 20 (3) (2012), 333-341.

[5] T. Jung and Q.H. Choi, Multiplicity results on a nonlinear biharmonic equation, Nonlinear Anal. TMA 30 (8) (1997), 5083-5092.

[6] A.C. Lazer and J.P. Mckenna, Multiplicity results for a class of semilinear elliptic and parabolic boundary value problems, J. Math. Anal. Appl. 107 (1985), 371395.

[7] A.M. Micheletti and A. Pistoia, Multiplicity results for a fourth-order semilinear elliptic problem, Nonlinear Anal. TMA 31 (7) (1998), 895-908.

[8] P.H. Rabinowitz, Minimax methods in critical point theory with applications to differential equations, CBMS-NSF Regional Conf. Ser. in Appl. Math. 65, Amer. Math. Soc., Providence, Rhode Island (1986).

Department of Mathematics Education

Inha University

Incheon 402-751, Korea

E-mail: qheung@inha.ac.kr

Department of Mathematics

Kunsan National University

Kunsan 573-701, Korea

E-mail: tsjung@kunsan.ac.kr 